\title{
Enhancing swimming and pumping performance of helical swimmers at low Reynolds numbers
}

\author{
Johan E. Quispe ${ }^{1,+}$, Aude Bolopion ${ }^{2}$, Pierre Renaud ${ }^{3}$ and Stéphane Régnier ${ }^{1}$
}

\begin{abstract}
Helical swimmers, actuated by low-strength uniform rotating magnetic fields, can develop swimming and pumping in low Reynolds number environments. They could play an important role for future in vitro and in vivo biomedical microrobotic tasks. Studying how their morphology influences swimming and pumping tasks is then of importance. In this paper, we focus on two geometrical aspects for optimizing both tasks: their helical shape and their cross-section. As a first contribution, we investigate the optimal performance of swimming and pumping. As a second contribution, we elucidate between optimal shapes at performing swimming and pumping at the same time. This study based on numerical simulation is intended to serve as a guiding reference for building optimal helical structures either for swimming, pumping, or a combination of both tasks.
\end{abstract}

Index Terms-Micro/Nano Robots, Automation at MicroNano Scales.

\section{INTRODUCTION}

W IRELESS microrobots are an attractive solution for microassembly [1], local remote sensing [2], and future medical interventions [3]. Due to their compactness and ease to actuate in very restrained environments, Helical Swimmers (HS) stand out for their common actuation method that requires low-strength uniform rotating magnetic fields of few milliteslas.

The importance of HS structures (HSs) relies on the functionalities they can display. The first is the swimming capability: HSs would be capable of navigating through low Reynolds $(R e)$ fluids in the human conduits to transport payloads such as drugs [4] or sperm cells [5]. The second functionality is pumping fluids in obstructed conduits, for mixing fluids [6] or dispensing microparticles [7]. Therefore, studying how their morphological changes affect their performance when realizing certain of such tasks is of great interest. We intend with this work, to provide guidelines for optimizing HSs for pumping and swimming.

Manuscript received: February, 24, 2021; Revised May, 22, 2021; Accepted June, 16, 2021.

This paper was recommended for publication by Editor Xinyu Liu upon evaluation of the Associate Editor and Reviewers' comments. This work is part of Multiflag project (ANR-16-CE33-0019) funded by the French National Research Agency (ANR) and supported by EIPHI Graduate School (Contract No. ANR-17-EURE-0002)

${ }^{+}$Corresponding author: quispedisir.upmc.fr

${ }^{1}$ ISIR, Sorbonne Univ., CNRS, Paris, France

${ }^{2}$ FEMTO-ST, UBFC, CNRS, Besançon, France

${ }^{3}$ ICube, Univ. of Strasbourg, INSA Strasbourg, Strasbourg, France

Digital Object Identifier (DOI): see top of this page.
A large number of studies on the helical swimming efficiency have been carried out experimentally $[8,9,10,11]$ and through simulations. In that regard, the versatility of numerical approaches was a key during optimization of geometrical aspects and different physical conditions.

Previous works based on boundary element method (BEM) have provided an important framework to find optimal helical structures at swimming. Several aspects such as helical shape variation $[12,13]$, cross-section orientation [14], degree of confinement [15] could be analysed. Other works with CFD have studied the effect on the swimming of different helical shapes on propulsion speed was explored [16]. In this paper, we address the effect of the cross-section and the helical shape to analyse their combined effect on swimming. Moreover, we provide an interesting framework to study helical swimming through two criteria concerning the type of magnetic source.

Regarding pumping, different aspects were studied such as the flux generation [15], thrust [17], viscoelastic media [18], and mixing capabilities [19]. On the other hand, efficiency at pumping has not been totally covered. Besides, the optimal operation of swimming and pumping at the same time has not been deeply covered in the literature. And, in the envisaged medical operations, swimmers might be required to perform both tasks in one mission e.g. to arrive at blood clots sites and apply a constant pumping action to reestablish the blood flow.

Numerical methods such as RFT and SBT methods are based on the partition of large bodies in segments that contribute independently to the total propulsion of the structure. Even if those techniques are computationally less expensive compared to BEM and CFD, aspects such as the shape or crosssection geometry, i.a. , cannot be analysed. In this work, we exploit CFD to model helical swimming and pumping in Newtonian fluids. This enables us to compute the generated flux at pumping as well as to investigate geometrical aspects that RFT and SBT methods cannot deal with e.g. low aspect ratios, small helical radius, and the cross-section elongation and orientation.

The formulation of the optimization problem is treated in section II. In section III and IV we analyse the different criteria for swimming and then for pumping. In section $\mathrm{V}$ we analyse the optimization of both tasks at the same time. Finally, we provide a summary of the relevant results obtained in this work in section VI. 


\section{FORMULATION OF THE OPTIMIZATION PROBLEM}

\section{A. Helical Shape}

We consider the following parametric equation $\psi$ to represent the helical centerline of HS structure (Fig. 1):

$$
\psi(s)=R \cos (s) \hat{\mathbf{x}}+R \sin (s) \hat{\mathbf{y}}+\frac{\lambda s}{2 \pi} \hat{\mathbf{z}}
$$

with $\psi: \mathbb{R} \rightarrow \mathbb{R}^{3}, s \in[0,2 \pi N], R$ the helical radius, $\lambda$ the pitch value, and $N$ the number of turns.

The HSs are considered initially to have a circular crosssection with radius $r$. To compare structures during the optimization, we set a constant total volume, $V=L \pi r^{2}$, with $L$ the total flagellum length. By doing that, we can reduce the number of possible geometrical configurations in Eq. (1). For that purpose, we introduce a dimensionless set of parameters $\left\{\beta, \theta, \frac{R}{r}\right\}$ with the aspect ratio $\beta=\frac{L}{2 r}$, the helical angle $\theta=\arcsin (\mathbf{t} \cdot \hat{z})$, and the normalized helical radius $\frac{R}{r}$. Note that the helical angle here is the complement of the pitch angle that is commonly defined as $\arccos (\mathbf{t} \cdot \hat{z})$ being $\mathbf{t}$ the tangent vector of the helix equation and $\hat{z}$ the propulsion direction of the swimmer. With those variables, Eq. (1) turns into:

$$
\begin{aligned}
\Lambda\left(\beta, \theta, \frac{R}{r}, s\right)= & \frac{R}{r}\left(\frac{V}{2 \pi \beta}\right)^{\frac{1}{3}} \cos (s) \hat{\mathbf{x}}+ \\
& \frac{R}{r}\left(\frac{V}{2 \pi \beta}\right)^{\frac{1}{3}} \sin (s) \hat{\mathbf{y}}+ \\
& \frac{R}{r}\left(\frac{V}{2 \pi \beta}\right)^{\frac{1}{3}} \tan (\theta) s \hat{\mathbf{z}}
\end{aligned}
$$

with $\theta \in\left[0, \frac{\pi}{2}\right], \frac{R}{r}$ and $\beta$ in $\mathbb{R}^{+}, s \in[0,2 \pi N]$, and $N=\frac{\beta \cos (\theta)}{\pi R / r}$. The cross-section is also parametrized for the optimization. We consider the general case of an elliptic geometry with major radius $a$, minor radius $b$, and orientation $\phi$ (Fig. 1(b) and (c)). Major and minor radius are computed considering the cross-section area as constant for each configuration with the same aspect ratio $\beta$, so, $a b=r^{2}$. By utilizing the vector base perpendicular to the helix centerline $\{\mathbf{n}, \mathbf{b}\}$ formed by the normal and binormal vectors, and the base associated to the minor and major axis respectively $\left\{\mathbf{n}^{\mathbf{p}}, \mathbf{b}^{\mathbf{p}}\right\}$, the orientation is defined as $\phi=\arccos \left(\mathbf{n} \cdot \mathbf{n}^{\mathbf{p}}\right)$. Thus, we use the ratio between major and minor radius $\frac{a}{b}$ and orientation $\phi$ as control parameters to tune the crosssection shape. The parameter $\phi$ has been already used in the literature for the building of twist-type HSs [20]. Then, its implementation in the design of future micromachines for medical purposes has been demonstrated to be practical and feasible.

\section{B. Magnetic Propulsion}

HSs are commonly actuated by low-strength uniform rotating magnetic fields. For that purpose, their composition include magnetic elements. They experience a magnetic

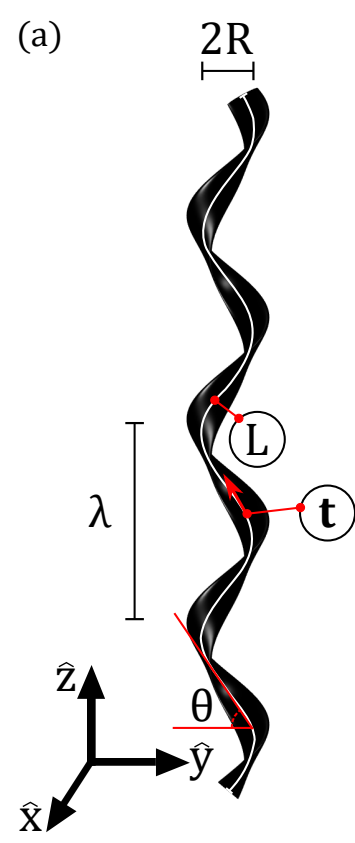

(b)

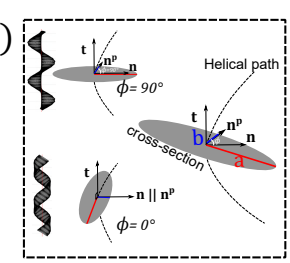

(c)

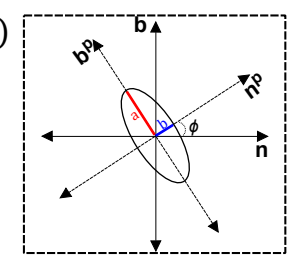

(d)

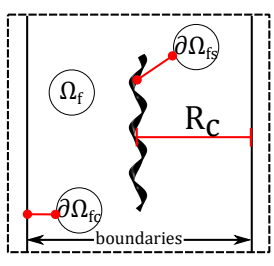

Figure 1: Geometrical parameters of HSs. (a) A general HS structure with elliptic cross-section showing the relevant parameters in our study namely, the helical angle $\theta$, the tangent to the helix centerline $\mathbf{t}$, the pitch $\lambda$, the flagellum length $L$, and the helical radius $R$. (b) Elliptic cross-section with major radius $a$, minor radius $b$ and orientation $\phi$. (c) Orientation of the cross-section $\phi$. (d) Notations: $\Omega_{f}$ is the fluid domain, $\partial \Omega_{f s}$ depicts boundaries of the HS in contact with the fluid, and $\partial \Omega_{f c}$ the container boundaries with radius $R_{c}$.

force $\mathbf{f}_{\mathbf{m}}$, and a magnetic torque $\tau_{\mathbf{m}}$ given by the following expressions [21]:

$$
\mathbf{f}_{\mathbf{m}}=V_{m}(\mathbf{m} \cdot \nabla) \mathbf{B} \quad \tau_{\mathbf{m}}=V_{m} \mathbf{m} \times \mathbf{B}
$$

where $V_{m}$ is the volume of the magnetic material presented in HSs, $\mathbf{m}$ is the volumic density of the magnetic moment, and $\mathbf{B}$ the magnetic field in which HSs are exposed. As the magnetic field is considered quasi-uniform [21], the magnetic force $\mathbf{f}_{\mathbf{m}}$ is negligible. Then, HSs only experience a magnetic torque $\tau_{\mathbf{m}}$ having a maximum value when $\mathbf{m} \perp \mathbf{B}$. Swimming and pumping depend on the magnetic torque $\tau_{\mathbf{m}}$, and at the same time $\tau_{\mathbf{m}}$ is affected by the magnetization method used to fabricate them. We have then to set conditions to investigate optimal swimming and pumping.

Regarding magnetization conditions, in [22], the magnetic material is deposited on the HSs' surface along the whole body length. Then, magnetic torque $\tau_{\mathbf{m}}$ for such cases depend on the surface of each structure. On the other hand, in [23, 24], authors made use of a soft-magnetic head prepared by e-beam evaporation. Hence, all structures experienced the same $\tau_{\mathbf{m}}$ under the same $\mathbf{B}$ but not the same fluidic torque $\tau_{\mathbf{f}}$ since they could have different geometries. Other methods combine the use of superparamagnetic polymer composites with two photon polymerization for the fabrication of HSs [20]. Through the application of an external magnetic field during the composite soft baking, 
(a)

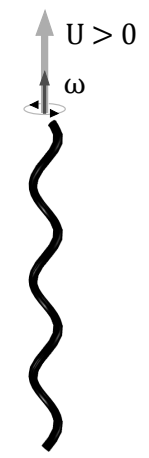

(b)

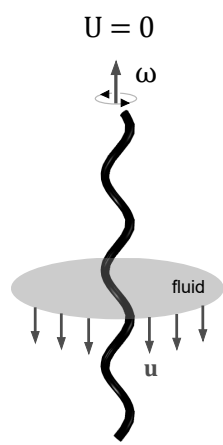

Figure 2: Different tasks of HSs. (a) Swimming. (b) pumping, in this task a tethered structure is considered $(U=0)$ and a flow is generated in the opposite direction of the thrust $(T)$.

the single-domain is magnetized parallel to the external magnetic field, achieving shape-independent magnetic properties. In this work, let us consider that all HSs have the same quantity of magnetic material $V_{m}$ as well as the volumic density of the magnetic moment $\mathbf{m}$. In that regard, the method used in [20] can apply for our subsequent evaluation. We consider structures with same volume that in average can keep the same quantity of magnetic material, if such particles are magnetized through the HS easy axis, in average, the magnetization of such structures will be almost the same. Other cases where this study could be applicable is when using magnetic heads [23], nonetheless, in such a case, the head drag have to be negligible to not alter the helical contribution to the thrust. Under such conditions, the differential factor for optimizing those structures relies on the fluidic torque $\tau_{\mathrm{f}}$ generated by them.

\section{Fluid and Helical Structure Dynamics}

HSs develop a rotary motion for swimming and pumping under low Reynolds number $R e$ conditions. The fluid flow generated by HS structure is then governed by NavierStokes (N-S) equations in the creeping flow regime given as follows:

$$
\begin{gathered}
\nabla \cdot \Gamma=0 \text { in } \Omega_{f} \times\left[0, t_{0}\right] \\
\Gamma=-p \mathbf{I}+\mu\left(\nabla \mathbf{u}+(\nabla \mathbf{u})^{T}\right) \\
\nabla \cdot \mathbf{u}=0 \text { in } \Omega_{f} \times\left[0, t_{0}\right]
\end{gathered}
$$

with $\mathbf{u}$ the fluid velocity field, $p$ the pressure, $\rho$ the fluid density and $\mu$ its dynamic viscosity. $\Gamma$ represents the stress on an infinitesimal fluid volume, $\Omega_{f}$ depicts the fluid domain (Fig. 1(d)), and $\left[0, t_{0}\right]$ the temporal domain. The boundary condition in the fluid-structure domain $\partial \Omega_{f s}$ are given by:

$$
\begin{gathered}
\mathbf{u}=\mathbf{u}_{s} \text { in } \partial \Omega_{f s} \times\left[0, t_{0}\right] \\
\mathbf{u}_{s}=\frac{d \mathbf{r}}{d t} \text { in } \partial \Omega_{f s} \times\left[0, t_{0}\right] \\
\mathbf{u}_{s}=\mathbf{U}+\omega \times\left(\mathbf{r}-\mathbf{r}_{\mathbf{0}}\right) \text { in } \partial \Omega_{f s} \times\left[0, t_{0}\right]
\end{gathered}
$$

where $\mathbf{u}_{s}$ is the HS structure velocity in one point $\mathbf{r}$ situated on its surface. $\mathbf{r}_{\mathbf{0}}$ is the HS center of mass, $\mathbf{U}=U \hat{z}$ is the propulsion swimming speed (cf. Fig. 2(a)), which in the case of pumping is zero (cf. Fig. 2(b)). Finally, $\omega=\omega \hat{z}$ is the HS angular velocity. The final boundary condition, defined for the limits of the container $\left(\partial \Omega_{f c}\right)$, is:

$$
\mathbf{u}=\mathbf{0} \text { in } \partial \Omega_{f c} \times\left[0, t_{0}\right]
$$

which indicates a zero fluid speed in the fluid-container boundary. Then, the fluidic force $\mathbf{f}_{\mathbf{f}}$ on the HS is computed by integrating the total stress $(\Gamma)$ over all the HS structure's surface.

$$
\mathbf{f}_{\mathbf{f}}=\int_{\partial \Omega_{f s}} \Gamma \mathbf{n}_{f} d s
$$

being $\mathbf{n}_{f}$ the normal vector to the surface of each finite element on the robot surface, and $d s$ the surface element.

Analogously, we can compute the fluidic torque $\tau_{\mathbf{f}}$ as follows:

$$
\tau_{\mathbf{f}}=\int_{\partial \Omega_{f s}}\left(\mathbf{r}-\mathbf{r}_{\mathbf{0}}\right) \times \Gamma \mathbf{n}_{f} d s
$$

Finally, as HSs are immersed in a low Re fluid, getting the steady state is quasi-immediate as the sum of external forces and torques is null:

$$
\mathbf{f}_{\mathbf{m}}+\mathbf{f}_{\mathbf{f}}=\mathbf{0} \quad \tau_{\mathbf{m}}+\tau_{\mathbf{f}}=\mathbf{0}
$$

\section{Criteria For Optimization}

Authors in [25] provides three different coefficients for evaluating the optimal swimming performance, namely, energetic efficiency, swimming efficiency, and propulsion efficiency. To compute those coefficients, they made use of the swimming speed $U$ (Fig. 2), the motor torque $\tau$ and drag coefficients given by RFT. A helical flagellum was then considered with a head as a base structure. Then, using the head drag coefficient $A_{0}$, the energy efficiency was computed as the ratio $\frac{A_{0} U}{\tau \omega}$. On the other hand, the drag effect was not considered for the definition of swimming efficiency, computed as the ratio $\frac{U}{\tau \omega}$. In [12] a dimensionless expression of swimming efficiency $\frac{\mu L^{2} U}{\tau}$ is proposed, with $\tau$ the fluidic torque and $L$ the flagellum length of the HS. In this work, we consider HSs without head or the case where the head drag can be negligible. Then, the latter expression is the most adapted. Swimming efficiency coefficient makes the optimization problem to be interpreted as to find the optimal structure that generates a greater speed consuming the least possible torque. On the other hand, the propulsion efficiency, defined as $\frac{U}{\omega}$, and can be regarded as a possible measurement of the linear distance covered per rotation.

Previous expressions, $\frac{\mu L^{2} U}{\tau}$ and $\frac{U}{\omega}$, stand for two different ways of focusing on the optimization problem considering the nature of the power source. The former definition indicates that we have a limited magnetic source, and in order to find an optimum HS structure, we have to search for structures that outperforms the others with the least possible torque. The latter definition refers to the fact that we have an infinite magnetic source, typically a few teslas, 

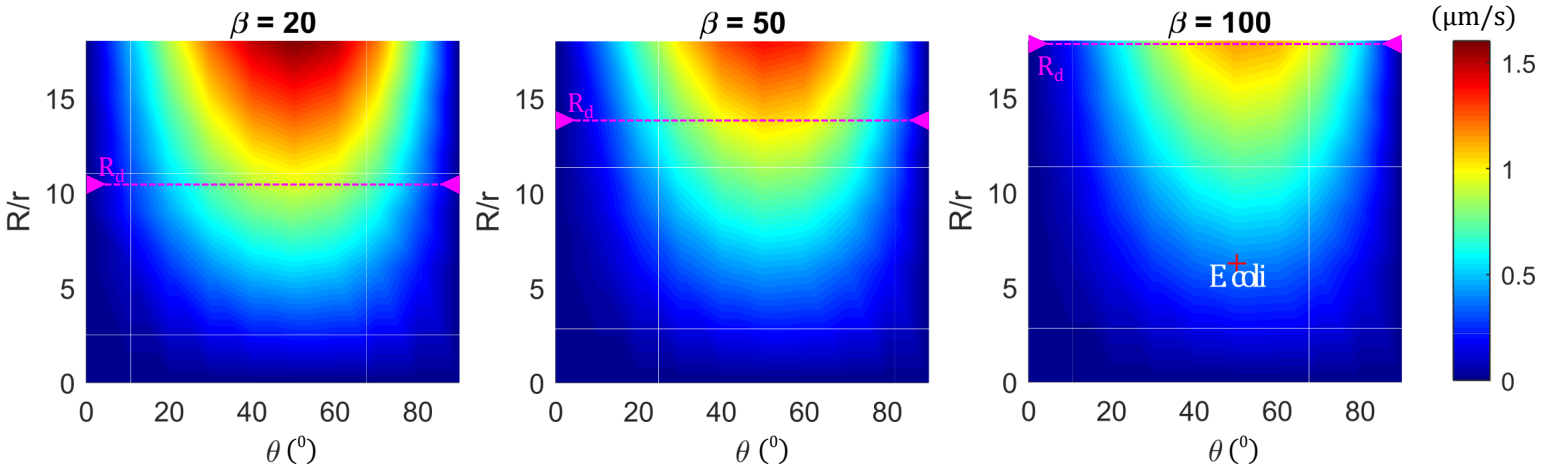

Figure 3: Color-map depicting the swimming speed (in $\mu \mathrm{m} / \mathrm{s}$ ) of structures with the same volume $V$ in the configuration space defined by $\left\{\beta, \theta, \frac{R}{r},\right\}$. To compute these results, HSs were set at constant angular speed $\omega=2 \pi H z$. $R_{d}$ represents a distance equivalent to $18 r_{100}=0.6318 \mu \mathrm{m}$, where $r_{100}=3.51 \times 10^{-2} \mu \mathrm{m}$ is the flagellum radius $r$ for the structure with aspect ratio $\beta=100$. Then, $18 r_{100} \approx 14.3 r_{50} \approx 10.5 r_{20}$.

which always provides the necessary torque to rotate the HS, thus, demonstrating no step-out frequencies or frequencies at which the magnetic torque $\tau_{\mathbf{m}}$ is not strong enough to rotate the HS structure synchronously with $\mathbf{B}$. Therefore, in such a case, evaluating the swimming speed per rotation indicates an optimum performance.

To evaluate pumping, in $[26,17]$ the total thrust $\left(T=\mathbf{f}_{\mathbf{f}} \cdot \hat{z}\right)$ generated by HSs is used as a study variable. In [15], authors introduce the total flux $I$ generated by HSs for studying pumping. They compute the flux $I=\int_{H} \mathbf{u} \cdot \mathbf{n}_{\mathbf{H}} d s$ in a plane $H$ perpendicular to axis of the HS, with $\mathbf{u}$ the fluid velocity field and $\mathbf{n}_{\mathbf{H}}$ the normal vector of plane $H$. In those cases, the pumping action is evaluated under the criterion of an infinite magnetic source. However, for future HS that must accomplish pumping and swimming tasks for one mission, the evaluation under the limited magnetic source criterion is of high importance.

The criteria used in our study consists in evaluating separately the swimming and pumping, considering the two types of magnetic sources namely, an infinite and a limited one. Afterward, we analyse the performance of both tasks at the same time.

For swimming, we evaluate propulsion efficiency with the situation of infinite magnetic source by proposing the following expressions as study variables: the swimming speed $U$ and the dimensionless speed $\frac{U}{L f}$ with $f=\frac{\omega}{2 \pi}$. Then, to evaluate the case where we account with a limited magnetic source, we use the dimensionless swimming efficiency $\frac{\mu L^{2} U}{\tau}$. For pumping, we treat the case of the infinite source through analysing directly the total thrust $T$ generated by HS structure and the normalized flux $\frac{I}{\omega R_{c}^{3}}$ at the middle plane of the HS $\left(H_{m}\right)$ with $R_{c}$ the container radius. To treat the case of the finite source we propose for the first time the dimensionless energetic efficiency at pumping expression, $\frac{T L}{\tau}$.

Finally, to consider at the same time swimming and pumping, we build an objective function $g$ that is the weighed sum of swimming and energetic efficiencies using a coefficient $\alpha \in[0,1]$ to indicate the balance between swimming and pumping priorities: $g=\alpha \frac{S}{S_{0}}+(1-\alpha) \frac{P}{P_{0}}$.

\section{SWIMMING PERFORMANCE OPTIMIZATION}

In this section, the fluid and structure dynamics is treated with the finite element method through a commercial software, COMSOL Multiphysics. For that purpose, we used the CFD-package with the Math package "arbitrary LagrangianEulerian" method (ALE) for treating the structure mesh displacement. Simulations were generated using a Intel(R) Core(TM) processor with 28GB-RAM. The mesh resolution was extremely fine (about 200000 elements on the HS surface). The computation time for each simulation in the transitory regime is between 5 and 25 min depending on the geometry of the HSs. The swimming of HSs is studied considering an angular speed $\omega$ of $2 \pi \mathrm{Hz}$, which is equivalent to 1 rotation per second. The choice of this value is appropriate since either flux and thrust have linear behaviours with respect to $\omega$. Afterwards, the choice of the constant volume $V$ was based on the flagellum bundle volume of the E. coli bacteria considered in $[27,15,28]$, the values of $L$ and $r$ are the ones considered in [15, 28]. Since $L$ and $R$ are $7.1 \mu \mathrm{m}$ and $0.035 \mu \mathrm{m}$ respectively, the computed volume is about $2.73 \times 10^{-20} \mathrm{~m}^{3}$, ensuring that the structures characteristic length will be in the microscale, Re can be computed as $\rho L U / \mu$, being in this study about $7.1 \times 10^{-6}$, the structures speed $U$ being about $1 \mu \mathrm{m} / \mathrm{s}$, and the fluid properties density $\rho$ and viscosity $\mu$ being that of distilled water.

\section{A. Helical Shape Influence}

Here, circular cross-sections are considered $\left(\frac{a}{b}=1\right)$. Figure 3 depicts the swimming speed $U$ of the different structures in the space of variables $\left\{\theta, \frac{R}{r}\right\}$ for aspect ratios $\beta=\{20,50,100\}$ corresponding to total lengths $L=$ $\{2.41,4.43,7.03\} \mu \mathrm{m}$ and flagellum radius $\left\{r_{20}=6.01, r_{50}=\right.$ $\left.4.43, r_{100}=3.51\right\} 10^{-8} \mathrm{~m}$, respectively. Optimum propulsion efficiency is achieved for HSs with a helical angle $\theta \in$ $\left[40^{\circ}, 60^{\circ}\right]$ and a normalized helical radius $\frac{R}{r}$ with the largest possible value. Knowing that the scale $\frac{R}{r}$ is different in each color-map for each aspect ratio $\beta$ depicted in Fig. 3, a fixed radial distance $R_{d}$ for each color-map will be situated 

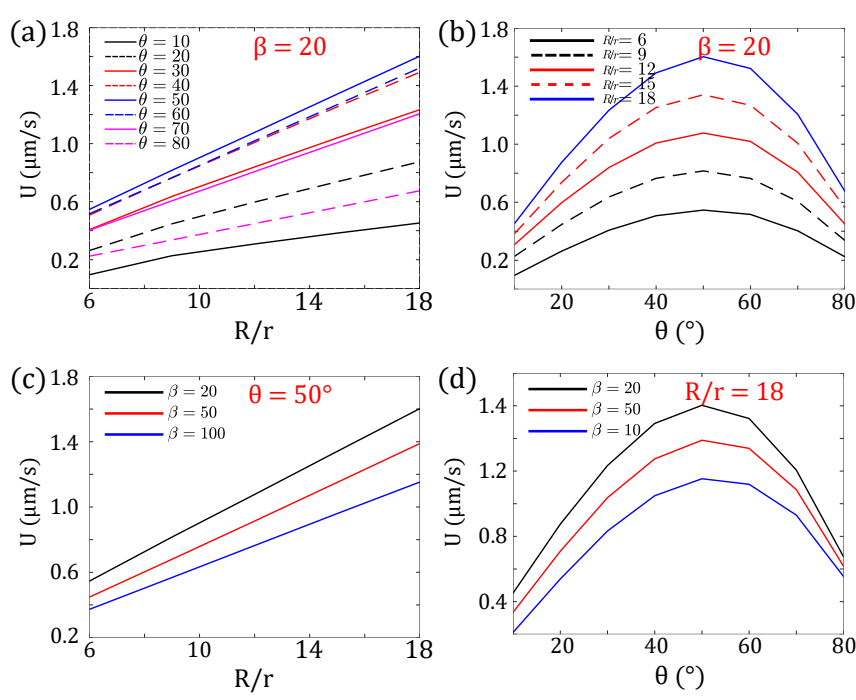

Figure 4: Dependence of the swimming propulsion speed $U$ on the configuration space set $\left\{\beta, \frac{R}{r}, \theta\right\}$. (a) $\frac{R}{r}$ vs. $U$ for $\theta \in[10,80]$ given $\beta=20$. (b) $\theta$ vs. $U$ for $\frac{R}{r} \in[6,18]$ given $\beta=20$. (c) $\frac{R}{r}$ vs. $U$ for $\beta \in\{20,50,100\}$ given $\theta^{r}=50^{\circ}$. (d) $\theta$ vs. $U$ for $\beta \in\{20,50,100\}$ given $\frac{R}{r}=18$.

differently. Here, we propose arbitrarily $R_{d}=18 r_{100}$ (when $\frac{R}{r}=18$ in graph with $\beta=100$ ) to observe the different positions that the same distance represents in all graphs. For the other color-map graphs, that distance represents other value in the scale $\frac{R}{r}$ e.g. $\frac{R}{r} \approx 14.3$ for graph with $\beta=50$ and $\frac{R}{r} \approx 10.5$ for graph with $\beta=20$ (Fig. 3 magenta line). $R_{d}$ can serve to establish a criterion for bounding the helical radius of HSs. Under that condition, HSs with $\beta=100$ can achieve a greater swimming speeds $U$ compared to HSs with lower aspect ratios. From these results, structures with larger aspect ratios and a helical angle $\theta \approx 50^{\circ}$ experiences a better propulsion efficiency. Fig. 4 represents the swimming speed $U$ with respect the set of variables $\left\{\beta, \frac{R}{r}, \theta\right\}$. Graphs (a) and (c) show a linear behavior of $U$ with respect to the normalized helical radius $\frac{R}{r}$. In addition, from graphs (b) and (d) a maximum value of $U$ is observed for $\theta \approx 50^{\circ}$. In order to have a better approximation, Fig. 5 proposes a zoom on the region of interest for $\beta=20$. In that figure the propulsion efficiency (a), the swimming efficiency (b), the fluidic torque (c), and the behaviour of $\frac{U}{L f}$ as function of $\theta(\mathrm{d})$ are depicted. Regarding the propulsion efficiency (cf. Fig. 5(a) and (d)), we found a maximum efficiency for $\theta=52.5^{\circ}$ for every $\frac{R}{r}$. In previous works [26, 29, 30], analytical models were implemented using RFT and SBT approximations. There exists a relation that links the propulsion speed with drag coefficients and the geometrical parameters of the helix given by $U=\omega R \frac{(\chi-1) \sin \left(2 \theta_{c}\right)}{2\left[1+(\chi-1) \sin ^{2}\left(\theta_{c}\right)\right]}$, with $\chi \approx 2$ using the slender body approximation. Through this assumption, the maximum speed is attained for $\theta_{c}=\frac{1}{2} \arccos \left(\frac{\chi-1}{\chi+1}\right)=35.26^{\circ}$, which is with our nomenclature equivalent to $\theta=54.74^{\circ}$. Nonetheless, we found a maximum angle for $\theta \approx 52.5^{\circ}$. The difference can be due to the slender-body approximation, that is not needed with finite element method. Structures are
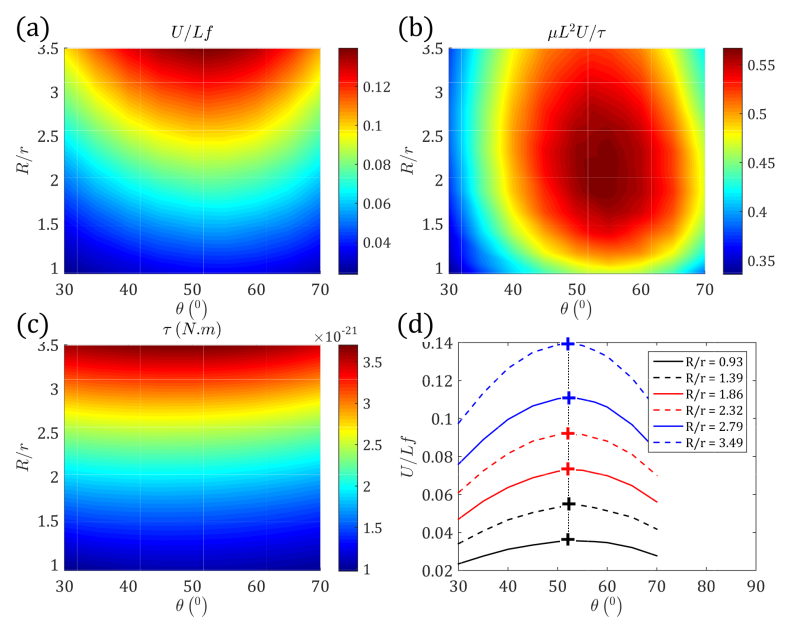

Figure 5: Optimization of HSs with aspect ratio $\beta=20$. (a) Performance per rotation $\left(\frac{U}{L f}\right)$. (b) Performance per torque $\left(\frac{\mu L^{2} U}{\tau}\right)$. (c) Fluidic torque $\tau$. (d) $\theta$ vs. $\frac{U}{L f}$ for different $\frac{R}{r}$ values.

then considered of quasi-zero filament width, and moreover, the force interaction with the neighboring points is neglected. Additionally, we considered structures with a small helical normalized radius $R / r$, which makes the contribution from the neighboring points much stronger. Experimental results in [20] are obtained with superparamagnetic HSs of regular composition and the same aspect ratio $\beta$ and helical normalized radius $R / r$. It is demonstrated that for $\theta=52.5^{\circ}$, the swimmer structures achieve a better dimensionless speed $U / L f$. Hence, our simulation results are in good agreement according to such experimental data. Regarding the torque, its value increases as $\frac{R}{r}$ increases (cf. Fig. 5(c)). On the other hand, regarding the normalized swimming efficiency, the maximum efficiency is found for $\theta=55^{\circ}$ and $\frac{R}{r}=2.3$ (cf. Fig. 5(b)). Keaveny et al. in [12] found the optimum swimmer under this criterion for $\theta=57.2^{\circ}$ and $\frac{R}{r}=2.2$. The slight differences between these two results can be explained by the fact that in [12] authors used BEM-based corrections for computing the traction and resistances coefficients of an RFT model, which provides a relationship of $\frac{U}{\tau}$ where parallel and normal drag coefficients are intervening. Here, we compute the kinematic and dynamic parameters of the HS using CFD by integrating the traction through solving Navier-stokes equations with the boundary conditions using finite elements, thus, dispensing with drag coefficients.

\section{B. Cross-Section Influence}

Here, we analyze the influence of cross-section parameters $\frac{a}{b}$ and $\phi$ on the propulsion efficiency. To do so, we choose arbitrarily a HS structure with $\beta=20, \theta=50^{\circ}$ and $\frac{R}{r}=3$. Fig. 6(a) represents the influence of the variables $\left\{\frac{a}{b}, \phi\right\}$ on the propulsion efficiency $\left(\frac{U}{L f}\right)$. The best performance are found for $\frac{a}{b}=4$ (the largest value) with $\phi=90^{\circ}$ that has an Archimedian pump-like structure with efficiency $\frac{U}{L f}=0.187$. The least performant is found for $\frac{a}{b}=4$ but $\phi=0$, having a ribbon like structure with efficiency $\frac{U}{L f}=0.078$. For 
(a)

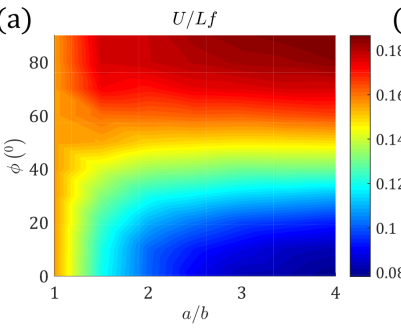

(c)

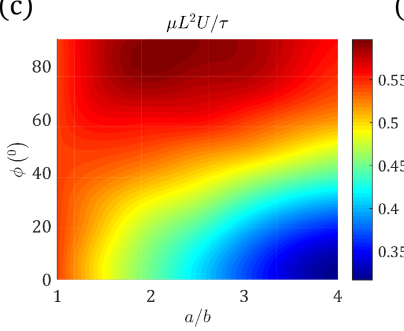

(e)

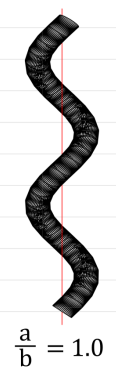

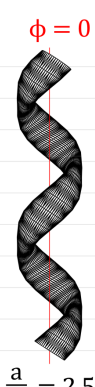

$\frac{\mathrm{a}}{\mathrm{b}}=2.5$ (b)

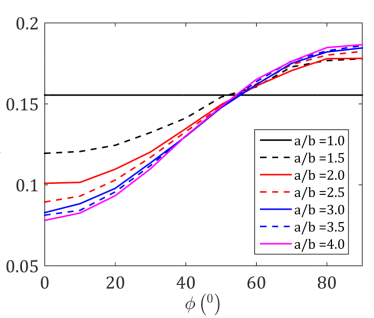

(d)

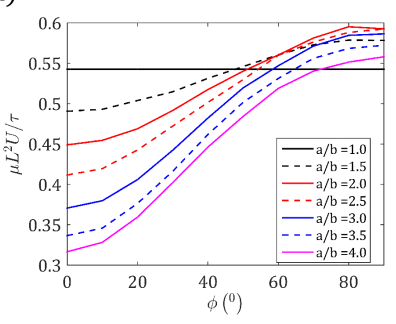

(f) Binor

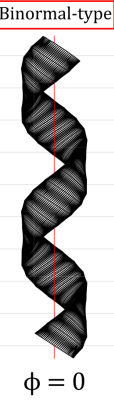

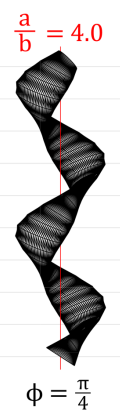

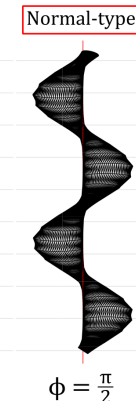

Figure 6: Cross-section influence of $\left\{\frac{a}{b}, \phi\right\}$ on the swimming performance of a HS with $\beta=20, \theta=50^{\circ}$ and $\frac{R}{r}=3$. (a) $\frac{U}{L f}$. (b) $\phi$ vs. $\frac{U}{L f}$ for different $\frac{a}{b}$. (c) $\frac{\mu L^{2} U}{\tau}$. (d) $\phi$ vs. $\frac{\mu L^{2} U}{\tau}$ for different $\frac{a}{b}$. (e) Cross-section deformation with respect to the ratio $\frac{a}{b}$ given $\phi=0$. (f) Change of the orientation $\phi$ given $\frac{a}{b}=4$, for $\phi=0$ and $\frac{\pi}{2}$ we have the binormal and normal-type respectively according to $[20]$.

structures with a circular cross-section, $\frac{a}{b}=1$, the efficiency is $\frac{U}{L f}=0.155$. If we compare the structures with the best and the one with the lowest performance, there is an increment on performance of $139 \%$. On the other hand, if we compare the structures with the best performance with the one which has a circular cross-section, it results in an increment of about $20 \%$ of propulsion efficiency. HSs in the literature having a binormal-type structure such as the ones presented in [24] at the micrometer scale, or in [31] at the millimeter scale could experience an increment of more than $100 \%$ in their propulsion speed if their cross-sections were twisted in $\phi=\frac{\pi}{2}$ becoming normal-type swimmers. Therefore, the studied structure with fixed helical parameters $(\beta=20$, $\theta=50^{\circ}$ and $\frac{R}{r}=3$ ) with an elongated cross-section $\frac{a}{b}=4$ and $\phi=90^{\circ}$ demonstrates the best swimming performance. Regarding results of HSs in a limited power source framework (Fig. 6(c) and (d)) the best performance is achieved for a HS with $a / b \approx 2.25$ and $\phi \approx 85^{\circ}\left(\frac{\mu L^{2} U}{\tau}=0.59\right)$ while the less performant as in the previous case is for a HS with the larger $a / b=4$ and $\phi=0^{\circ}\left(\frac{\mu L^{2} U}{\tau}=0.32\right)$. Such novel result would impact future designs of future HS. Fig. 6(e)
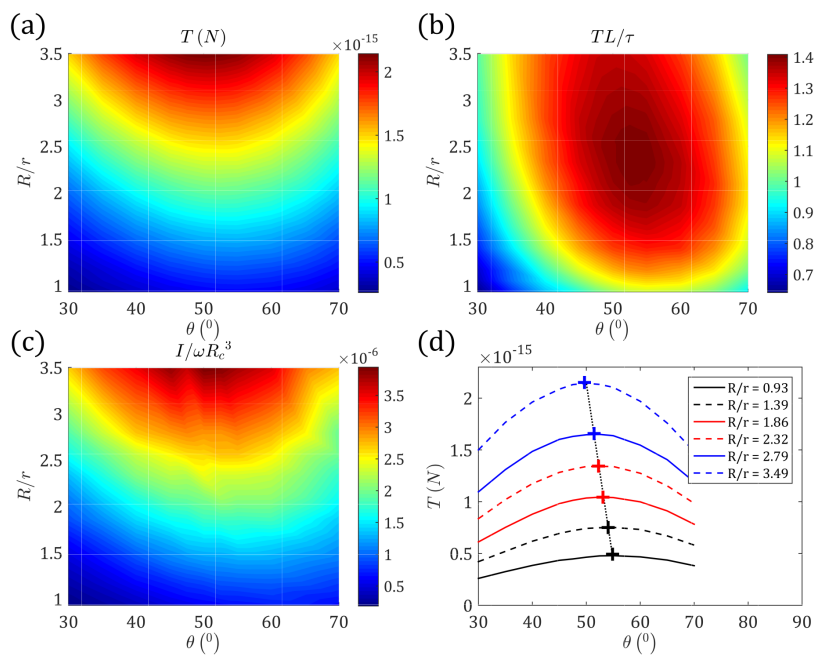

Figure 7: Influence of the helical shape on pumping for HSs with $\beta=20$. (a) Thrust at pumping fixed angular speed $\omega$ for a variable space defined by $\left\{\theta, \frac{R}{r}\right\}$. Normalized thrust/torque $\frac{T L}{\tau}$ (energetic efficiency). (c) Normalized flux $\frac{I}{\omega R_{c}^{3}}$. (d) Thrust as a function of $\theta$ for different $\frac{R}{r}, \theta$ optimum changes for each $\frac{R}{r}$.

and (f) depicts the HS morphology as a function of $\frac{a}{b}$ and $\phi$.

\section{PUMPING PERFORMANCE IMPROVEMENT}

The pumping of HSs is investigated by first studying how the helical shape influences the pumping performance and then analyse the effect of the cross-section.

\section{A. Helical shape influence}

Here, we study HSs with circular cross-sections with an arbitrary value of aspect ratio $\beta=20$. Fig. 7(a) represents the thrust at pumping generated in the variable space defined by $\left\{\theta, \frac{R}{r}\right\}$. The helical angle $\theta$ that maximises thrust is function of $\frac{R}{r}$ (cf. Fig. 7(d)) i.e. $\theta_{\max }=55.0^{\circ}$ for $\frac{R}{r}=0.9$, $\theta_{\max }=52.5^{\circ}$ for $\frac{R}{r}=2.3$, and $\theta_{\max }=50.0^{\circ}$ for $\frac{R}{r}=3.5$. On the other hand, Fig. 7(b) depicts the energetic efficiency at pumping $\frac{T L}{\tau}$. The optimal parameters are found for $\theta=55^{\circ}$ and $\frac{R}{r}=2.3$. Figure 7(c) stands for the normalized flux $\frac{I}{\omega R_{S}^{3}}$ generated at the middle cross-sectional plane $H_{m}$ of the $\mathrm{HS}$ structure. The maximum $\frac{I}{\omega R_{c}^{3}}$ is generated by helical angles $\theta$ ranging from $\left[45^{\circ}, 50^{\circ}\right]$.

\section{B. Cross-section influence}

For the analysis of the cross-section influence, we choose as we did in section III. $B$, a HS structure with parameters $\beta=20, \theta=50^{\circ}$ and $\frac{R}{r}=3$. Fig. 8(a-b) represents the influence of the thrust at pumping, the maximum value obtained is for $\frac{a}{b}=4$ (the largest value) and $\phi=90^{\circ}$ with $T=30.9 \times 10^{-15} \mathrm{~N}$. The least performant one is achieved by the structure whose parameters are $\frac{a}{b}=4$ and $\phi=0$ with $T=12.1 \times 10^{-15} \mathrm{~N}$. The best structure outperforms in $156 \%$ the least performant. Moreover, a structure with 
circular cross-section generates a thrust at pumping of about $T=18.0 \times 10^{-15} \mathrm{~N}$. Then, the best structure with respect to this last one increases the thrust $71.9 \%$. Fig. 8(c-d) represents the energetic efficiency at pumping $\frac{T L}{\tau}$. Similarly to the previous results, the parameters that maximises the efficiency are found for $\frac{a}{b}=4$ and $\phi=90^{\circ}$ with $\frac{T L}{\tau}=1.65$, and the parameters for the least performant are $\frac{a}{b}=4$ and $\phi=0$ with $\frac{T L}{\tau}=0.83$. In this case, the relative increment is about $99 \%$ with respect to the least performant structure. With respect to the structures with a circular cross-section, $\frac{T L}{\tau}=1.38$, this increment is about $20 \%$. Analogously, it happens the same when analysing the normalized flux $\left(\frac{I}{\omega R_{c}^{3}}\right)$ (cf. Fig. 8(e-f)). The parameters that maximises $\frac{I}{\omega R_{c}^{3}}$ are found for large values of $\frac{a}{b}$ with $\phi \sim 80-90^{\circ}$.

\section{SWIMMING AND PUMPING ENHANCEMENT}

\section{A. Criterion}

To assess the HSs that can provide efficient swimming and pumping, it is needed first to define our optimization framework. Firstly, we define a dimensionless function $g$ that links both efficiencies, at swimming and pumping, respectively. This function is defined as follows:

$$
g(\mathbf{x}, \alpha)=\alpha \frac{S(\mathbf{x})}{S_{0}}+(1-\alpha) \frac{P(\mathbf{x})}{P_{0}}
$$

where $S(\mathbf{x})=\frac{\mu L^{2} U(\mathbf{x})}{\tau(\mathbf{x})}$ is the efficiency at swimming and $S_{0}$ is the maximum reached value. The efficiency at pumping is $P(\mathbf{x})=\frac{T(\mathbf{x}) L}{\tau(\mathbf{x})}$ and the maximum value reached $P_{0}$. Note that $\mathbf{x}=\left(\beta, \theta, \frac{R}{r}\right)$. The balance between swimming and pumping importance is obviously dependent on the exact applicative context. To handle this, the parameter $\alpha$ indicates which task is prioritized i.e. $\alpha=0$ for pumping task while $\alpha=1$ for swimming. The optimization problem is thus defined as follows: $\max g(\mathbf{x}, \alpha)$ subject to $V=$ const. \& $\beta=20$ with $\theta \in\left[30^{\circ}, 70^{\circ}\right], \frac{R}{r} \in[0.9,3.5]$ and $\alpha \in[0,1]$.

\section{B. Results}

From Fig. 9, we can observe the slight evolution of the darker region (optimal zone) as a function of $\alpha$. We made distinction between 3 different regions, i.e. for $g=0.90$, 0.95 and 0.98 . Those are highlighted with thicker color lines in Fig. 9. It is interesting to see that a wide region exists $(R / r \in 1.8-2.7, \theta \in 48-58)$ where swimming and pumping are possible with a high efficiency $(g=0.98)$. On the other hand, the optimum configuration is unmodified when considering swimming, pumping, or their combination. The global optimum parameters in all cases are found for $\theta=55^{\circ}$ and $\frac{R}{r}=2.3$. Those results, which are novel to our knowledge, shows it is possible to have optimal performance for swimming and pumping using a single geometry of HS belonging to a wide region in the parameter space. This is very interesting for the design of versatile HS in future applicative contexts.
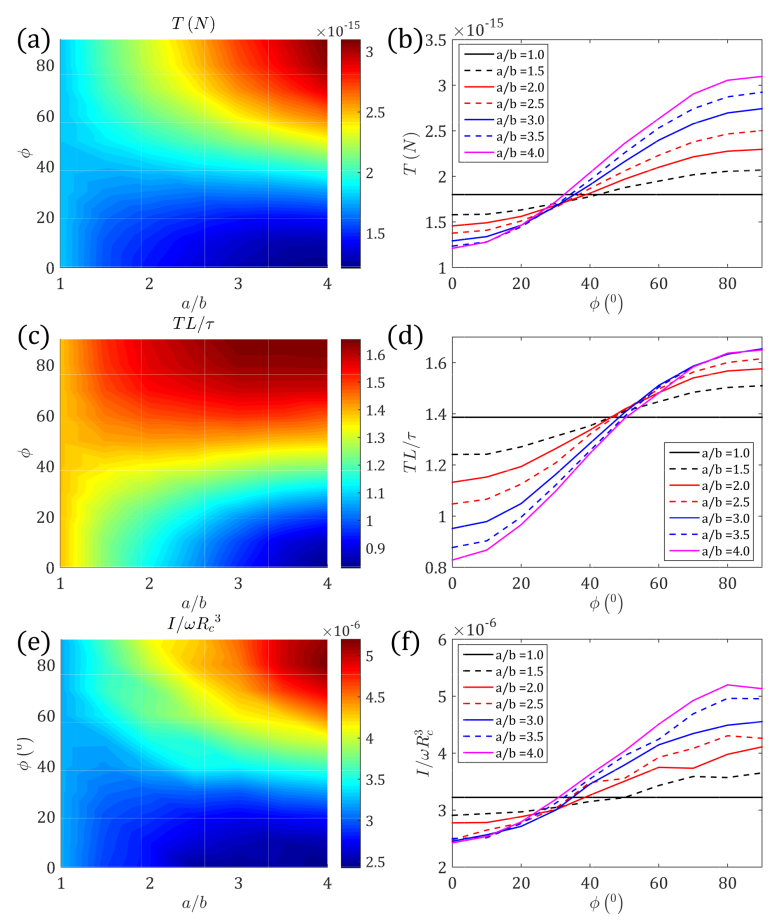

Figure 8: Influence of the cross-section at pumping. (a-b) Thrust as a function of the variable space $\left\{\frac{a}{b}, \phi\right\}$. (c-d) Normalized thrust per torque $\left(\frac{T L}{\tau}\right)$. (e-f) Normalized flux $\left(\frac{I}{\omega R_{c}^{3}}\right)$.

\section{CONCLUSIONS}

Through this study, we review different criteria for maximising efficiency of helical swimmer structures (HSs) for two specific tasks namely, pumping and swimming. We mainly investigated two geometrical features concerning the helical shape through the variable space defined by the aspect ratio, the helical angle and the normalized helical radius, $\left\{\beta, \theta, \frac{R}{r}\right\}$, and the cross-section shape via the elongation and the cross-section orientation $\left\{\frac{a}{b}, \phi\right\}$. We observed that structures with a certain elongation $\frac{a}{b}>1$ can achieve a relative high efficiency per rotation at swimming and pumping if their cross-sections are oriented with $\phi=90^{\circ}$. In the limited power source case, we found the existence of an optimum for $\frac{a}{b} \approx 2.25$ and $\phi \approx 85^{\circ}$. We identified optimal structure for pumping and swimming, defined by $\theta=55^{\circ}$, $\frac{R}{r}=2.3$ under the definition of efficiency per torque, limited power source case. In addition, it was observed through the analysis of a criterion combining swimming and pumping performance that the same geometry is of interest for combined tasks. This study is intended to serve as a guiding reference for building optimal HSs for future medical tasks, which is the main perspective of this work. One first next step of this study is to test the optimal designs through experiments. Moreover, in envisioned medical interventions, HSs must navigate through non-Newtonian bio-fluids with viscoelastic behavior that may alter the dynamics of helical robots. In that regard, our next step toward such in-vivo applications will be to study HS optimization under such complex conditions. 

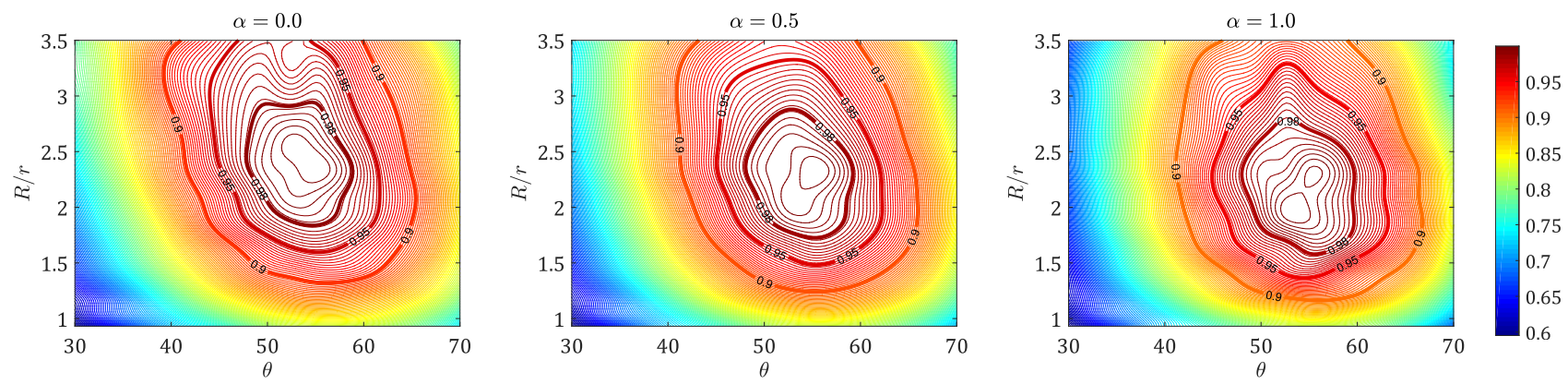

Figure 9: Optimization of swimming and pumping at the same time. The solutions are depicted for $\alpha=0$ (just pumping), $\alpha=0.50$ (half relevance on swimming and half on pumping), and $\alpha=1.00$ (just swimming).

\section{REFERENCES}

[1] T.-Y. Huang, F. Qiu, H.-W. Tung, K. E. Peyer, N. Shamsudhin, J. Pokki, L. Zhang, X.-B. Chen, B. J. Nelson, and M. S. Sakar, "Cooperative manipulation and transport of microobjects using multiple helical microcarriers," Rsc Advances, vol. 4, no. 51, pp. 26771$26776,2014$.

[2] A. Ghosh, D. Dasgupta, M. Pal, K. I. Morozov, A. M. Leshansky, and A. Ghosh, "Helical nanomachines as mobile viscometers," Advanced Functional Materials, vol. 28, no. 25, p. 1705687, 2018.

[3] B. J. Nelson, I. K. Kaliakatsos, and J. J. Abbott, "Microrobots for minimally invasive medicine," Annual review of biomedical engineering, vol. 12, pp. 55-85, 2010.

[4] F. Qiu, S. Fujita, R. Mhanna, L. Zhang, B. R. Simona, and B. J. Nelson, "Magnetic helical microswimmers functionalized with lipoplexes for targeted gene delivery," Advanced Functional Materials, vol. 25, no. 11, pp. 1666-1671, 2015.

[5] M. Medina-Sánchez, L. Schwarz, A. K. Meyer, F. Hebenstreit, and O. G. Schmidt, "Cellular cargo delivery: Toward assisted fertilization by sperm-carrying micromotors," Nano letters, vol. 16, no. 1, pp. 555-561, 2015.

[6] M. J. Kim and K. S. Breuer, "Use of bacterial carpets to enhance mixing in microfluidic systems," Journal of Fluids Engineering, vol. 129, no. 3, p. 319-324, 2007.

[7] T.-Y. Huang, M. S. Sakar, A. Mao, A. J. Petruska, F. Qiu, X.-B. Chen, S. Kennedy, D. Mooney, and B. J. Nelson, "3d printed microtransporters: Compound micromachines for spatiotemporally controlled delivery of therapeutic agents," Advanced Materials, vol. 27, no. 42, pp. 6644-6650, 2015.

[8] T. Xu, G. Hwang, N. Andreff, and S. Régnier, "Modeling and swimming property characterizations of scaled-up helical microswimmers," IEEE/ASME Transactions on Mechatronics, vol. 19, no. 3, pp. 10691079, 2013.

[9] T. Xu, J. Yu, C.-I. Vong, B. Wang, X. Wu, and L. Zhang, "Dynamic morphology and swimming properties of rotating miniature swimmers with soft tails," IEEE/ASME Transactions on Mechatronics, vol. 24, no. 3, pp. 924-934, 2019.

[10] J. Quispe and S. Régnier, "Magnetic miniature swimmers with multiple rigid flagella," in 2020 IEEE International Conference on Robotics and Automation (ICRA), 2020, pp. 9237-9243.

[11] J. E. Quispe, A. Oulmas, and S. Régnier, "Geometry optimization of helical swimming at low reynolds number," in 2019 International Conference on Manipulation, Automation and Robotics at Small Scales (MARSS), 2019, pp. 1-6.

[12] E. E. Keaveny, S. W. Walker, and M. J. Shelley, "Optimization of chiral structures for microscale propulsion," Nano letters, vol. 13, no. 2, pp. 531-537, 2013.

[13] J. Miao, X. Li, B. Liang, J. Wang, and X. Xu, "Enhancing swimming performance by optimizing structure of helical swimmers," Sensors, vol. 21, no. 2, p. 494, 2021.

[14] E. E. Keaveny and M. J. Shelley, "Applying a second-kind boundary integral equation for surface tractions in stokes flow," Journal of Computational Physics, vol. 230, no. 5, pp. 2141-2159, 2011.

[15] B. Liu, K. S. Breuer, and T. R. Powers, "Propulsion by a helical flagellum in a capillary tube," Physics of Fluids, vol. 26, no. 1, p. 011701, 2014.
[16] A. Acemoglu and S. Yesilyurt, "Effects of geometric parameters on swimming of micro organisms with single helical flagellum in circular channels," Biophysical journal, vol. 106, no. 7, pp. 1537-1547, 2014.

[17] S. Zhong, K. W. Moored, V. Pinedo, J. Garcia-Gonzalez, and A. J. Smits, "The flow field and axial thrust generated by a rotating rigid helix at low reynolds numbers," Experimental thermal and fluid science, vol. 46, pp. 1-7, 2013.

[18] L. Li and S. E. Spagnolie, "Swimming and pumping by helical waves in viscous and viscoelastic fluids," Physics of Fluids, vol. 27, no. 2, p. $021902,2015$.

[19] A. Buchmann, L. J. Fauci, K. Leiderman, E. Strawbridge, and L. Zhao, "Mixing and pumping by pairs of helices in a viscous fluid," Physical Review E, vol. 97, no. 2, p. 023101, 2018.

[20] C. Peters, O. Ergeneman, P. D. W. García, M. Müller, S. Pané, B. J. Nelson, and C. Hierold, "Superparamagnetic twist-type actuators with shape-independent magnetic properties and surface functionalization for advanced biomedical applications," Advanced Functional Materials, vol. 24, no. 33, pp. 5269-5276, 2014.

[21] X. Wang, C. Hu, S. Pane, and B. J. Nelson, "Dynamic modeling of magnetic helical microrobots," IEEE Robotics and Automation Letters, 2021.

[22] A. Ghosh and P. Fischer, "Controlled propulsion of artificial magnetic nanostructured propellers," Nano letters, vol. 9, no. 6, pp. 2243-2245, 2009.

[23] D. J. Bell, S. Leutenegger, K. Hammar, L. Dong, and B. J. Nelson, "Flagella-like propulsion for microrobots using a nanocoil and a rotating electromagnetic field," in Proceedings 2007 IEEE international conference on robotics and automation. IEEE, 2007, pp. 1128-1133.

[24] L. Zhang, J. J. Abbott, L. Dong, B. E. Kratochvil, D. Bell, and B. J. Nelson, "Artificial bacterial flagella: Fabrication and magnetic control," Applied Physics Letters, vol. 94, no. 6, p. 064107, 2009.

[25] N. Giuliani, L. Heltai, and A. DeSimone, "Predicting and optimizing microswimmer performance from the hydrodynamics of its components: The relevance of interactions," Soft robotics, vol. 5, no. 4, pp. 410-424, 2018.

[26] O. Raz and J. Avron, "Swimming, pumping and gliding at low reynolds numbers," New Journal of Physics, vol. 9, no. 12, p. 437, 2007.

[27] S. Koyasu and Y. Shirakihara, "Caulobacter crescentus flagellar filament has a right-handed helical form," Journal of molecular biology, vol. 173, no. 1, pp. 125-130, 1984.

[28] N. C. Darnton, L. Turner, S. Rojevsky, and H. C. Berg, "On torque and tumbling in swimming escherichia coli," Journal of bacteriology, vol. 189, no. 5, pp. 1756-1764, 2007.

[29] J. Yang, C. W. Wolgemuth, G. Huber, et al., "Kinematics of the swimming of spiroplasma," Physical review letters, vol. 102, no. 21, p. 218102, 2009.

[30] K. I. Morozov and A. M. Leshansky, "The chiral magnetic nanomotors," Nanoscale, vol. 6, no. 3, pp. 1580-1588, 2014.

[31] T. Xu, G. Hwang, N. Andreff, and S. Régnier, "Influence of geometry on swimming performance of helical swimmers using doe," Journal of Micro-Bio Robotics, vol. 11, no. 1, pp. 57-66, 2016. 